\title{
Association of elevated inflammatory and endothelial biomarkers with prehypertension among Mongolians in China
}

\author{
Guiyan Wang ${ }^{1,2}$, Aili Wang ${ }^{1}$, Weijun Tong ${ }^{1}$, Yanbin Liu ${ }^{1}$ and Yonghong Zhang ${ }^{1}$
}

Chronic inflammation and endothelial dysfunction may be associated with hypertension and cardiovascular disease. We examined associations between inflammatory and endothelial dysfunction biomarkers and the risk of prehypertension among Mongolians. A cross-sectional study was conducted among 2589 Mongolians aged 20 years and older in Inner Mongolia, China. Three blood pressure measurements, body weight, height and lifestyle factors were obtained for all participants. Overnight fasting blood samples were obtained to measure the biomarkers, including C-reactive protein (CRP), soluble intercellular adhesion molecule-1 (sICAM-1), soluble E-selectin (sE-selectin) and angiotensin II. The average levels of CRP (7.43 vs. 5.86), sICAM-1 (339.38 vs. 328.05), sE-selectin (19.11 vs. 18.32) and angiotensin II (52.00 vs. 47.00) were all significantly higher in hypertensives than that in prehypertensives (all $P<0.05)$; prehypertensives had higher levels of CRP (5.86 vs. 4.85) and sICAM-1 (328.05 vs. 314.14) compared with normotensives (both $P<0.05$ ). Hypertension (odds ratio (OR): $1.50,95 \%$ confidence interval $(\mathrm{Cl}): 1.07,2.11$ ) and prehypertension (OR: $1.38,95 \% \mathrm{Cl}: 1.02,1.85$ ) were positively and significantly associated with elevated CRP adjusted for multivariable. Hypertension (OR: $1.56,95 \% \mathrm{Cl}: 1.18,2.06$ ) and prehypertension (OR: $1.32,95 \% \mathrm{Cl}: 1.02,1.71$ ) were also positively and significantly associated with higher sICAM-1 adjusted for age and gender. Elevated CRP and sICAM-1 were associated with prehypertension among Mongolian population. This study suggests that inflammation and endothelial dysfunction may have a role in the development of hypertension.

Hypertension Research (2011) 34, 516-520; doi:10.1038/hr.2010.255; published online 23 December 2010

Keywords: endothelial dysfunction; inflammation; prehypertension

\section{INTRODUCTION}

Hypertension is a serious public health problem in the world because of its high prevalence and consequently increased risk of cardiovascular disease. As the Seventh Report of the Joint National Committee on the Prevention, Detection, Evaluation, and Treatment of High Blood Pressure (JNC7) ${ }^{1}$ defined prehypertension as a systolic blood pressure (SBP) of $120-139 \mathrm{~mm} \mathrm{Hg}$ and/or a diastolic blood pressure (DBP) of $80-89 \mathrm{~mm} \mathrm{Hg}$, some researchers focused interest on the relationship between prehypertension and cardiovascular disease. Although prehypertension has not currently been categorized as a disease, some evidence have documented that people with prehypertension have more cardiovascular risk factors, ${ }^{2}$ increased risks of hypertension ${ }^{3}$ and cardiovascular events compared with those with normal $\mathrm{BP}^{4-6}$

Inflammation and endothelial dysfunction are novel risk factors for atherosclerosis and clinical cardiovascular disease. ${ }^{7}$ Several previous studies have showed that an elevated level of C-reactive protein (CRP) is associated with increased risk of hypertension. ${ }^{8-10}$ Several studies reported that CRP, soluble intercellular adhesion molecule-1
(sICAM-1) and soluble E-selectin (sE-selectin) were related to prehypertension. ${ }^{1-14}$ However, most of these studies were conducted in Western populations. The association of inflammation and endothelial dysfunction with prehypertension has been poorly studied in Asian populations.

In the present study, we examine the association between plasma levels of CRP, sICAM-1, sE-selectin and angiotensin II and the risk of prehypertension in a Mongolian population from China.

\section{METHODS}

Study participants

We conducted a cross-sectional study between 2002 and 2003 in Inner Mongolia, an autonomous region in north China that borders Mongolia and Russia. The detailed methods for the study participant selection and data collection are presented elsewhere. ${ }^{15}$ Briefly, the study participants aged 20 years and older were recruited from 32 villages in 2 adjacent townships located in Kezuohou Banner (county) and Naiman Banner in Inner Mongolia. The majority of local residents were Mongolians who had lived there for many generations and maintained a traditional diet and lifestyle. The study

${ }^{1}$ Department of epidemiology, School of Radiation Medicine and Public Health, Medical College of Soochow University, Suzhou, PR China and ${ }^{2}$ Department of Health Care, Xiamen Maternal and Child Care Hospital, Xiamen, PR China

Correspondence: Dr Y Zhang, Department of Epidemiology, School of Radiation Medicine and Public Health, Medical College of Soochow University, 199 Renai Road, Industrial Park District, Suzhou 215123, PR China.

E-mail: yhzhang@suda.edu.cn

Received 29 July 2010; revised 5 November 2010; accepted 7 November 2010; published online 23 December 2010 
population consisted of both farmers and herdsmen whose diets were high in fat and salt. There were a total of 3475 Mongolian people aged 20 and older living in these villages; among them, 2589 persons participated in the study.

This study was approved by the Soochow University Radiation Medicine and Public Health School institutional review board. Written informed consent was obtained from all study participants.

\section{Data collection}

Data on demographic information, lifestyle risk factors, family history of hypertension and personal medical history were obtained using a standard questionnaire administered by trained staff. Cigarette smoking was defined as having smoked at least one cigarette per day for 1 year or more. The information regarding amount and type of alcohol consumed during the past years was collected, and alcohol drinking was defined as consuming at least $50 \mathrm{~g}$ distillate spirits ( $\sim 50 \%$ alcohol concentrate, namely, $25 \mathrm{~g}$ alcohol) per day for 1 year or more.

Three sitting BP measurements were taken by trained observers using a standard mercury sphygmomanometer according to a standard protocol, after the subjects had been resting for $30 \mathrm{~min}$, and there were $30 \mathrm{~s}$ intervals between two BP measurements. The first and fifth Korotkoff sounds were recorded as SBP and DBP, respectively. The mean of the three BP measurements was used in the analysis. Hypertension was defined as SBP $\geqslant 140 \mathrm{~mm} \mathrm{Hg}$ and/or DBP $\geqslant 90 \mathrm{~mm} \mathrm{Hg}$ and/or use of antihypertensive medication in recent 2 weeks. Participants who had mean SBP/or DBP within the range of 120-139/ $80-89 \mathrm{~mm} \mathrm{Hg}$ and have never been told that they have hypertension were defined as prehypertensives. ${ }^{1}$ Body height and weight, waist circumference and hip circumference were measured by trained staff using a balance beam scale after subjects removed their shoes and were wearing light clothing. Body mass index (BMI) was calculated as the weight in kilograms divided by the square of the height in meters. Waist circumference and hip circumference were measured at the level of $1 \mathrm{~cm}$ above the umbilicus. Waist circumference and hip circumference ratio (WHR) was calculated as the ratio of waist circumference to hip circumference. For this analysis, high BMI was defined as $\geqslant 24 \mathrm{~kg} \mathrm{~m}^{-2}$ and high WHR was defined as $\geqslant 0.9$ based on the recommendations of the Working Group on Obesity in China. ${ }^{16}$

Blood samples were collected in the morning after at least $8 \mathrm{~h}$ of fasting. All plasma and serum samples were frozen at $-80^{\circ} \mathrm{C}$ until laboratory testing. Fasting plasma glucose (FPG) was measured using a modified hexokinase enzymatic method; hyperglycemia was defined as FPG $\geqslant 6.1 \mathrm{mmoll}^{-1} .{ }^{17}$ Concentrations of total cholesterol (TC), high-density lipoprotein-cholesterol and triglycerides (TGs) were assessed enzymatically on a Beckman Synchrony CX5 Delta Clinical System (Beckman Coulter, Fullerton, CA, USA) using commercial reagents, and low-density lipoprotein-cholesterol (LDL-C) concentration was calculated by means of the Friedewald equation for participants who had less than $400 \mathrm{mg}$ per $100 \mathrm{ml}$ TGs. ${ }^{18}$ Dyslipidemia was defined as TC $\geqslant$ $5.72 \mathrm{mmoll}^{-1}$ and/or TG $\geqslant 1.69 \mathrm{mmoll}^{-1}$ and/or LDL-C $\geqslant 3.64 \mathrm{mmoll}^{-1}$ and/or high-density lipoprotein-cholesterol $<0.91 \mathrm{mmoll}^{-1} .{ }^{19} \mathrm{CRP}$ was determined by an immunoturbidimetric assay on a Beckman Synchron CX5 Delta Clinical System using commercial reagents. sE-selectin and sICAM-1 were measured by an enzyme-linked immunosorbent assay assay (R \& D Systems, Minneapolis, MN, USA) that employs the quantitative sandwich enzyme immunoassay technique. Angiotensin II concentration was measured by radioimmunoassay after separation by high-performance liquid chromatography.

\section{Statistical analysis}

The unadjusted means and s.d.s of continuous variables and prevalence of categorical variables were calculated for hypertensive, prehypertensive and normotensive individuals, respectively. For the biomarkers, the mean and s.d. of sICAM-1 were calculated for the three groups because of normal distribution; comparison between the three groups was conducted by using analysis of variance models. Medians and interquartile ranges of CRP, sE-selectin and angiotensin II were calculated for the three groups because of non-normal distribution; comparison between the three groups was conducted by using Wilcoxon rank-sum test.

To assess whether inflammation or endothelial dysfunction was associated with prehypertension and hypertension, logistic regression was used to calculate the odds ratio (OR) for prehypertension and hypertension associated with elevated plasma concentrations of each biomarker, respectively. The dependent variable (prehypertension or hypertension) and independent variables were binary, and elevated plasma concentrations of biomarkers were defined as a measurement in the upper quartile of the values for this study population. The first logistic regression model included only biomarker; age and gender were added in the second model. In the third model, BMI, WHR, FPG, smoking, alcohol drinking, LDL-C, TC, TG and family history of hypertension were also included in the analysis. All $P$-values were two tailed, and a significance level of 0.05 was used. Statistical analysis was conducted using SAS statistical software (version 9.1; SAS Institute Inc, Cary, NC, USA).

\section{RESULTS}

Baseline characteristics of Mongolians according to BP status

A total of 2589 participants aged 20-84 years, including 1064 men and 1525 women, were included in the analysis. There were 968 (37.39\%) hypertensives, 994 (38.39\%) prehypertensives and 627 (24.22\%) normotensives among the participants. Baseline characteristics of study participants according to BP status are shown in Table 1 . The results showed that the hypertensives tended to have older age and greater BMI, WHR, FPG, TG, TC and LDL-C levels, and higher rates of male, family history of hypertension, cigarette smoking, alcohol drinking compared with prehypertensives and normotensives $(P<0.05)$. Prehypertensives likely had older age and greater BMI, WHR, FPG, TG, TC and LDL-C levels, and higher rates of male, family history of hypertension, alcohol drinking compared with normotensives $(P<0.05)$. There were no statistical differences for high-density lipoprotein-cholesterol concentrations among the three groups $(P>0.05)$.

Inflammatory and endothelial biomarkers according to BP status The median (interquartile range) for CRP, sE-selectin and angiotensin II, and the mean (s.d.) of sICAM-1 are shown in Table 2. Levels of CRP, sE-selectin, angiotensin II and sICAM-1 were higher in

\section{Table 1 Baseline characteristics of 2589 Mongolians according to BP status in Mongolia, China}

\begin{tabular}{lccc}
\hline Factors & Normotensives & Prehypertensives & Hypertensives \\
\hline$N$ & 627 & 994 & 968 \\
Age (year) & $41.07 \pm 10.61$ & $44.55 \pm 11.78^{*}$ & $52.03 \pm 12.05^{*, \#}$ \\
Male (\%) & 27.11 & $41.15^{*}$ & $50.10^{*, \#}$ \\
Smoking (\%) & 40.99 & 42.86 & $48.24^{*, \#}$ \\
Alcohol drinking (\%) & 20.10 & $31.29^{*}$ & $44.32^{*, \#}$ \\
FHH (\%) & 3.83 & $7.55^{*}$ & $24.69^{*, \#}$ \\
BMI (kg m$\left.{ }^{-2}\right)$ & $21.35 \pm 2.94$ & $22.03 \pm 3.28^{*}$ & $23.05 \pm 3.79^{*, \#}$ \\
WHR & $0.84 \pm 0.06$ & $0.86 \pm 0.06^{*}$ & $0.89 \pm 0.07^{*, \#}$ \\
SBP (mm Hg) & $106.62 \pm 7.63$ & $121.70 \pm 7.85^{*}$ & $153.08 \pm 24.18^{*, \#}$ \\
DBP (mm Hg) & $70.89 \pm 5.52$ & $80.85 \pm 4.34^{*}$ & $97.18 \pm 10.62^{*, \#}$ \\
FPG (mmol I-1) & $4.70 \pm 1.01$ & $4.92 \pm 1.02^{*}$ & $5.25 \pm 1.44^{*, \#}$ \\
TC (mmol I-1) & $3.45 \pm 0.99$ & $3.69 \pm 1.08^{*}$ & $3.98 \pm 1.22^{* \#}$ \\
TG (mmol I-1) & $0.80(0.55,1.14)$ & $0.92(0.65,1.35)^{*}$ & $1.12(0.87,1.77)^{*, \#}$ \\
HDL-C (mmol I-1) & $1.16 \pm 0.31$ & $1.18 \pm 0.34$ & $1.17 \pm 0.33$ \\
LDL-C (mmol I-1) & $1.99(1.49,2.55)$ & $2.16(1.60,2.81)^{*} 2.33(1.72,3.13)^{*, \#}$
\end{tabular}

Abbreviations: BMI, body mass index; DBP, diastolic blood pressure; $\mathrm{FHH}$, family history of hypertension; FPG, fasting plasma glucose; HDL-C, high-density lipoprotein-cholesterol; IQR, interquartile range; LDL-C, low-density lipoprotein-cholesterol; SBP, systolic blood pressure; TC, total cholesterol; TG, triglyceride; WHR, waist-to-hip ratio.

For normally distributed variable, values are presented as means (s.d.), and $P$-value was computed with analysis of variance test for the difference in mean; for non-normally distributed variables, values are presented as medians (IQR) and $P$-values were computed using Wilcoxon test for the difference in medians; for categorical variables, $P$-values were computed using $\chi^{2}$-test for the difference in prevalence.

$\chi^{2}$-test for the difference in prevalence.

\#Compared with prehypertensives: $P<0.05$. 
Table 2 Mean (s.d.) or median (IQR) of inflammatory and endothelial biomarkers according to BP status

\begin{tabular}{lcrr}
\hline Factors & Normotensives & Prehypertensives & Hypertensives \\
\hline CRP $\left(\mathrm{mgl}^{-1}\right)$ & $4.85(3.30-8.14)$ & $5.86(3.85-10.75)^{*}$ & $7.43(4.57-15.07)^{*, \#}$ \\
sICAM-1 (ng ml & $328.05 \pm 98.22^{*}$ & $339.38 \pm 99.13^{*, \#}$ \\
sE-selectin $\left(\mathrm{ng} \mathrm{ml}^{-1}\right)$ & $314.14 \pm 93.67$ & $18.32(14.78-24.26)$ & $19.11(15.37-25.48)^{*, \#}$ \\
Angiotensin II $\left(\mathrm{pg} \mathrm{ml}^{-1}\right)$ & $17.93(13.78-24.43)$ & $47.00(40.00-67.20)$ & $52.00(40.50-83.80)^{*, \#}$ \\
\hline
\end{tabular}

Abbreviations: CRP, C-reactive protein; IQR, interquartile range; sE-selectin, soluble E-selectin; sICAM-1, soluble intercellular adhesion molecule-1.

For normally distributed variable (sICAM-1), values are presented as means (s.d.), and $P$-value was computed with analysis of variance test for the difference in mean; for non-normally distributed

variables (CRP, sE-selectin and angiotensin II), values are presented as medians (IQR), and $P$-values were computed using Wilcoxon test for the difference in medians.

${ }^{*}$ Compared with normotensives: $P<0.05$.

"Compared with prehypertensives: $P<0.05$.

Table 3 ORs and $95 \%$ Cls of prehypertension and hypertension associated with biomarkers of inflammation and endothelial dysfunction

\begin{tabular}{|c|c|c|}
\hline Variables & OR (95\% Cl) of prehypertension & OR $(95 \%$ Cl) of hypertension \\
\hline \multicolumn{3}{|l|}{$C R P\left(m g l^{-1}\right)$} \\
\hline Model $1^{a}$ & $1.89(1.44,2.47)$ & $3.16(2.42,4.11)$ \\
\hline Model $2^{b}$ & $1.71(1.29,2.25)$ & $2.70(2.02,3.60)$ \\
\hline Model $3^{c}$ & $1.38(1.02,1.85)$ & $1.50(1.07,2.11)$ \\
\hline \multicolumn{3}{|c|}{ Quartiles of ICAM-1 (ng m/ $\left.{ }^{-1}\right)$} \\
\hline Model $1^{a}$ & $1.33(1.04,1.72)$ & $1.75(1.36,2.24)$ \\
\hline Model $2^{b}$ & $1.32(1.02,1.71)$ & $1.56(1.18,2.06)$ \\
\hline Model $3^{c}$ & $1.20(0.92,1.57)$ & $1.17(0.85,1.60)$ \\
\hline \multicolumn{3}{|c|}{ Quartiles of E-selectin $\left(n g m l^{-1}\right)$} \\
\hline Model $1^{a}$ & $0.98(0.77,1.24)$ & $1.14(0.90,1.45)$ \\
\hline Model $2^{b}$ & $0.90(0.70,1.14)$ & $0.98(0.75,1.28)$ \\
\hline Model $3^{c}$ & $0.83(0.64,1.07)$ & $0.77(0.57,1.04)$ \\
\hline \multicolumn{3}{|c|}{ Quartiles of angiotensin // (pg m/ $\left.{ }^{-1}\right)$} \\
\hline Model $1^{a}$ & $1.14(0.89,1.46)$ & $1.73(1.36,2.20)$ \\
\hline Model $2^{b}$ & $1.13(0.88,1.45)$ & $1.71(1.30,2.24)$ \\
\hline Model $3^{c}$ & $1.14(0.88,1.48)$ & $1.65(1.22,2.23)$ \\
\hline \multicolumn{3}{|c|}{$\begin{array}{l}\text { Abbreviations: BMI, body mass index; } \mathrm{Cl} \text {, confidence interval; FPG, fasting plasma glucose; } \\
\text { CRP, C-reactive protein; ICAM-1, intercellular adhesion molecule-1; LDL-C, low-density } \\
\text { lipoprotein-cholesterol; OR, odds ratio; TC, total cholesterol; TG, triglyceride; WHR, } \\
\text { waist-to-hip ratio. } \\
\text { aOnly biomarkers were included as independent variables. } \\
\text { bAdjusted for age and gender. } \\
\text { cAdjusted for age, gender, BMI, WHR, FPG, smoking, alcohol drinking, LDL-C, TC, TGs and } \\
\text { family history of cardiovascular disease. }\end{array}$} \\
\hline
\end{tabular}

hypertensives than those in prehypertensives or normotensives, respectively $(P<0.05)$. The prehypertensives also have higher CRP and sICAM-1 levels compared with normotensives $(P<0.05)$. There was no statistical difference for $\mathrm{sE}$-selectin and angiotensin II levels between prehypertensives and normotensives $(P>0.05)$.

ORs of prehypertension and hypertension associated with biomarkers of inflammation and endothelial dysfunction

The ORs of prehypertensives and hypertensives associated with elevated biomarkers of inflammation and endothelial dysfunction are presented in Table 3. Compared with normotensives, elevated CRP (all $P<0.05$ in three models) and angiotensin II (all $P<0.05$ in three models) were associated with increased risk of hypertension, and elevated sICAM-1 was associated with increased risk of hypertension under unadjusted for other factors or after adjusted for age and gender (both $P<0.05$ in models 1 and 2). Inversely, hypertension was not significantly associated with increased risk of elevated sE-selectin ( $P>0.05$ for three models).
Similarly, compared with normotensives, elevated CRP (all $P<0.05$ in three models) was associated with increased risk of prehypertension, and elevated sICAM-1 was associated with increased risk of prehypertension under unadjusted for other factors or after adjusted for age and gender (both $P<0.05$ in models 1 and 2). Inversely, prehypertension was not significantly associated with increased risk of elevated sE-selectin or angiotensin II ( $P>0.05$ for three models). ORs of prehypertension were weaker than those of hypertension.

\section{DISCUSSION}

In this cross-sectional study among a Mongolian population, we found that plasma levels of CRP and sICAM-1 were positively and significantly associated with hypertension and prehypertension. Individuals with higher levels of CRP and sICAM-1 probably were at an increased risk of hypertension and prehypertension compared with those with normal levels of CRP and sICAM-1. These data provide further support that inflammation and endothelial dysfunction may have a role in the development from prehypertension to hypertension.

Several prospective cohort studies have indicated that CRP levels were predictive of the risk of myocardial infarction, ischemic stroke and vascular death. ${ }^{20}$ There was also evidence to suggest that CRP may have a direct role as a mediator in atherogenesis by inducing intracellular adhesion molecule expression. ${ }^{7,21}$ Our previous study and other studies showed that CRP may be an independent risk factor for hypertension. ${ }^{8-10}$ Sesso et al. $^{22}$ reported that elevated CRP was associated with an increased risk of incidence of hypertension in a cohort study that included people with baseline BP in the prehypertension range. Possible mechanisms for this association include oxidative stress ${ }^{23}$ and interactions with adhesion molecules, plasminogen activator inhibitor-1 and LDL-C uptake..$^{21,24,25}$

Data of NHANES III $^{11}$ showed that prehypertensives had a higher prevalence of elevated CRP $\left(>3.0 \mathrm{mgl}^{-1}\right)$ than normotensives (27.4 vs. 19.8\%). After adjustment for multivariable, participants with SBP of $120-139 \mathrm{~mm} \mathrm{Hg}$ or DBP of $80-89 \mathrm{~mm} \mathrm{Hg}$ were more likely to have elevated CRP than those with SBP $<120 \mathrm{~mm} \mathrm{Hg}$ or DBP $<80 \mathrm{~mm} \mathrm{Hg}$. Their findings were consistent to ours, in which elevated CRP associated with increased risk of hypertension (OR: 1.50, 95\% confidence interval $(\mathrm{CI}): 1.07,2.11)$ and prehypertension (OR: 1.38, 95\% CI: 1.02, 1.85), respectively, adjusted for multivariable.

Chrysohoou's study ${ }^{12}$ revealed an association between prehypertension and inflammatory markers (CRP, WBC counts, tumor necrosis factor- $\alpha$, amyloid-a and homocysteine) among cardiovascular diseasefree adults, independent of other coexisting risk factors or unhealthy lifestyle behavior, suggesting that prehypertension might be a proinflammatory condition. In this work, inflammation seems to have a critical role in the development of hypertension, as these prehypertensives with increased CRP seem to be more vulnerable in developing hypertension during the 5 years of follow-up. 
In our relatively large population-based study, plasma levels of sICAM-1, but not sE-selectin and angiotensin II, were significantly higher in prehypertensives than that in normotensives. In addition, we only found the rude OR and age- and gender-adjusted OR of prehypertension for higher sICAM-1 were statistically significant. Our findings were similar to DeSouza's findings, in which 11 hypertensive subjects had higher plasma levels of sICAM-1 and soluble vascular cell adhesion molecule-1 but not sE-selectin compared with 10 normotensive controls, ${ }^{26}$ and univariate analysis revealed a positive correlation between sICAM-1 and both SBP $(r=0.50, P=0.02)$ and DBP $(r=0.49, P=0.03)$. Hlubocka reported that 22 hypertensive patients had significantly higher levels of sICAM-1 but not soluble vascular cell adhesion molecule-1, sE-selectin and soluble P-selectin compared with 22 normotensive subjects. ${ }^{27}$

In an observational study in Taiwan, ${ }^{13}$ there were 20 prehypertensives and 41 hypertensives without other risk factors (for example, diabetes mellitus, dyslipidemia and obesity) and 17 normotensive controls. Hypertensive patients had significantly higher circulating levels of sICAM-1 compared with normotensive controls $(P=0.009)$. The mean sICAM-1 level was significantly higher in the prehypertensives than that in the normotensive controls $(P=0.03)$. No significant differences in levels of soluble vascular cell adhesion molecule-1 and sE-selectin were found between the two groups. In a communitybased study including 664 men and women living in England, Miller et al. reported that plasma level of sE-selectin was significantly associated with elevated BP in women but not in men. ${ }^{14}$ Additionally, sICAM-1 was not significantly associated with elevated BP in either women or men. The relationship between biomarkers of inflammation and endothelial dysfunction and elevated BP varies with gender, age and abdominal obesity, ${ }^{3,14}$ which may partially explain previous inconsistencies in the literature. In our analysis, we adjusted for these confoundings to estimate the ORs of hypertension or prehypertension for the biomarkers. Under adjusted for only age and gender, sICAM-1 was significantly associated with both hypertension and prehypertension; the OR of both hypertension or prehypertension for sICAM-1 tended to be statistically significant because those low limits of $95 \%$ CI for OR were near to 1 , although the ORs did not reach level of significance after adjusting for multivariate. Whether adjusted for only age and gender or multivariate, the ORs of both hypertension and prehypertension for CRP were statistically significant. Therefore, we believe that there is probably a certain extent of inflammation and endothelial dysfunction in the phase of prehypertension; inflammation and endothelial dysfunction may promote prehypertension progressing to hypertension.

Besides its role as a vasoconstrictor, angiotensin II also induces endothelial dysfunction and inflammation, resulting in accelerated progression of atherosclerosis. ${ }^{28,29}$ Angiotensin II may have a direct role in inducing hypertension by increasing oxidative stress and inflammation. ${ }^{30,31}$ In our study, an increased angiotensin II level was found among individuals with hypertension but not prehypertension.

The present study found that concentrations of all the four biomarkers of inflammation and endothelial dysfunction in hypertensives were significantly higher than that in prehypertensives, and that CRP and sICAM-1 of the four biomarkers of inflammation and endothelial dysfunction in prehypertensives were significantly higher than that in normotensives; the ORs of hypertension associated with the biomarkers were significantly greater than the ORs of prehypertension associated with the biomarkers. These findings indicated that the progression to hypertension from prehypertension could be the results of gradual accumulation of inflammatory reaction and endothelial dysfunction, and accumulation of some traditional cardiovascular risk factors. In previous paper, ${ }^{15}$ we have reported that prevalence of some traditional cardiovascular risk factors and number of the risk factors in prehypertensives are similar to that in hypertensives, but weaker than hypertensives, which imply that prehypertension had the same risk factors as hypertension and some cardiovascular risk factors have existed in the stage of prehypertension.

Several limitations of this study should be mentioned. First, this was a cross-sectional study, therefore a causal relationship between inflammatory and endothelial biomarkers and the risk of hypertension could not be established. Secondly, $\sim 25 \%$ of the eligible population from these villages did not choose to participate, which may have introduced some selection bias. However, we believe this bias is minimal because it is unlikely that participants did not choose to participate because of their BP or biomarkers levels that they did not know. Furthermore, several important biomarkers of inflammation and endothelial dysfunction were not measured in our study. There were several important strengths of our study that deserve to be mention too. This is the largest study to examine the association between the biomarkers of inflammation and endothelial dysfunction and hypertension or prehypertension in an Asian population. The study participants were homogeneous regarding their genetic background and environmental exposures. The study data were collected with rigid quality control, and important covariables were measured and controlled in analysis.

In conclusion, our study indicated that higher plasma levels of CRP and slCAM-1 were associated with not only hypertension but also prehypertension. These data support the hypotheses that inflammation and endothelial dysfunction might have an important role in the etiology of hypertension. Future prospective cohort studies and clinical trials should be conducted to test the causal relationship between inflammation and endothelial dysfunction and the risk of hypertension.

\section{CONFLICT OF INTEREST}

The authors declare no conflict of interest.

\section{ACKNOWLEDGEMENTS}

We are deeply appreciative of the participants in the study, and thank Kezuohouqi Banner Center for Disease Prevention and Control, and Naiman Banner Center for Disease Prevention and Control for their support and assistance. This study was supported by the National Natural Science Foundation of China (Grant Nos. 30471484 and 30972531).

1 Chobanian AV, Bakris GL, Black HR, Cushman WC, Green LA, Izzo JL, Jones DW, Materson BJ, Oparil S, Wright JT, Roccella EJ, Natl High Blood Pressure Educ P. The Seventh Report of the Joint National Committee on Prevention, Detection, Evaluation, and Treatment of High Blood Pressure: the JNC 7 Report. JAMA-J Am Med Assoc 2003; 289: 2560-2572.

2 Greenlund KJ, Croft JB, Mensah GA. Prevalence of heart disease and stroke risk factors in persons with prehypertension in the United States, 1999-2000. Arch Intern Med 2004; 164: 2113-2118.

3 Pitsavos C, Chrysohoou C, Panagiotakos DB, Lentzas Y, Stefanadis C. Abdominal obesity and inflammation predicts hypertension among prehypertensive men and women: the ATTICA Study. Heart Vessels 2008; 23: 96-103.

4 Qureshi AI, Suri MFK, Kirmani JF, Divani AA, Mohammad Y. Is prehypertension a risk factor for cardiovascular diseases? Stroke 2005; 36: 1859-1863.

5 Liszka HA, Mainous AG, King DE, Everett GJ, Egan BM. Prehypertension and cardiovascular morbidity. Ann Fam Med 2005; 3: 294-299.

6 Kshirsagar AV, Carpenter M, Bang H, Wyatt SB, Colindres RE. Blood pressure usually considered normal is associated with an elevated risk of cardiovascular disease. $\mathrm{Am} \mathrm{J}$ Med 2006; 119: 133-141. 
7 Szmitko PE, Wang CH, Weisel RD, de Almeida JR, Anderson TJ, Verma S. New markers of inflammation and endothelial cell activation: part I. Circulation 2003; 108: 1917-1923.

8 Zhang YH, Thompson AM, Tong WJ, Xu T, Chen J, Zhao L, Kelly TN, Chen CS, He J. Biomarkers of inflammation and endothelial dysfunction and risk of hypertension among Inner Mongolians in China. J Hypertens 2010; 28: 35-40.

9 Sung KC, Suh JY, Kim BS, Kang JH, Kim H, Lee MH, Park JR, Kim SW. High sensitivity C-reactive protein as an independent risk factor for essential hypertension. Am J Hypertens 2003; 16: 429-433.

10 Lakoski SG, Herrington DM, Siscovick DM, Hulley SB. C-reactive protein concentration and incident hypertension in young adults: the CARDIA study. Archives of Internal Medicine 2006; 166: 345-349.

11 King DE, Egan BM, Mainous III AG, Geesey ME. Elevation of C-reactive protein in people with prehypertension. J Clin Hypertens (Greenwich) 2004; 6 : 562-568.

12 Chrysohoou C, Pitsavos C, Panagiotakos DB, Skoumas J, Stefanadis C. Association between prehypertension status and inflammatory markers related to atherosclerotic disease: the ATTICA Study. Am J Hypertens 2004; 17: 568-573.

13 Hwang YS, Tsai WC, Lu YH, Lin CC, Tsai KY. Effects of angiotensin II-receptor blockers on soluble cell adhesion molecule levels in uncomplicated systemic hypertension: an observational, controlled pilot study in Taiwanese adults. Curr Ther Res Clin Exp 2005; 66: 181-194.

14 Miller MA, Kerry SM, Cook DG, Cappuccio FP. Cellular adhesion molecules and blood pressure: interaction with sex in a multi-ethnic population. J Hypertens 2004; 22: 705-711.

15 Li HM, Xu T, Tong WJ, Liu YB, Zhao L, Zhang YH. Comparison of cardiovascular risk factors between prehypertension and hypertension in a Mongolian population, Inner Mongolia, China. Circ J 2008; 72: 1666-1673.

16 Bei-Fan Z, Cooperative Meta Anal Grp W. Predictive values of body mass index and waist circumference for risk factors of certain related diseases in Chinese adults: study on optimal cut-off points of body mass index and waist circumference in Chinese adults. Asia Pac J Clin Nutr 2002; 11: S685-S693.

17 Alberti KG, Zimmet PZ. Definition, diagnosis and classification of diabetes mellitus and its complications. Part 1: diagnosis and classification of diabetes mellitus provisional report of a WHO consultation. Diabet Med 1998; 15: 539-553.

18 Friedewald WT, Levy RI, Fredrickson DS. Estimation of the concentration of low-density lipoprotein cholesterol in plasma, without use of the preparative ultracentrifuge. Clin Chem 1972; 18: 499-502.
19 Fang Q, Wang ZL, Ning TH, Shao G, Chen ZJ, Lu ZL. Suggestions of prevention and treatments for dyslipidemia (in Chinese). Chinese J Cardiol 1997; 25: 169-175.

20 Ridker PM. C-reactive protein and the prediction of cardiovascular events among those at intermediate risk: moving an inflammatory hypothesis toward consensus. J Am Coll Cardiol 2007; 49: 2129-2138.

21 Pasceri V, Willerson JT, Yeh ETH. Direct proinflammatory effect of C-reactive protein on human endothelial cells. Circulation 2000; 102: 2165-2168.

22 Sesso HD, Buring JE, Rifai N, Blake GJ, Gaziano JM, Ridker PM. C-reactive protein and the risk of developing hypertension. JAMA J Am Med Assoc 2003; 290: 2945-2951.

23 Yasunari K, Maeda K, Nakamura M, Yoshikawa J. Oxidative stress in leukocytes is a possible link between blood pressure, blood glucose, and C-reacting protein. Hypertension 2002; 39: 777-780.

24 Pasceri V, Chang J, Willerson JT, Yeh ETH. Modulation of C-reactive protein-mediated monocyte chemoattractant protein-1 induction in human endothelial cells by antiatherosclerosis drugs. Circulation 2001; 103: 2531-2534.

25 Devaraj S, Xu DY, Jialal I. C-reactive protein increases plasminogen activator inhibitor-1 expression and activity in human aortic endothelial cells. Circulation 2003; 107: 398-404.

26 DeSouza CA, Dengel DR, Macko RF, Cox K, Seals DR. Elevated levels of circulating cell adhesion molecules in uncomplicated essential hypertension. Am J Hypertens 1997; 10: 1335-1341.

27 Hlubocka Z, Umnerova V, Heller S, Peleska J, Jindra A, Jachymova M, Kvasnicka J, Horky K, Aschermann M. Circulating intercellular cell adhesion molecule-1, endothelin-1 and von Willebrand factor-markers of endothelial dysfunction in uncomplicated essential hypertension: the effect of treatment with ACE inhibitors. J Hum Hypertens 2002; 16: 557-562.

28 Schiffrin EL, Touyz RM. Editorial comment. Multiple actions of angiotensin II in hypertension: benefits of AT(1) receptor blockade. J Am Coll Cardiol 2003; 42: 911-913.

29 Pastore L, Tessitore A, Martinotti S, Toniato E, Alesse E, Bravi MC, Ferri C, Desideri G, Gulino A, Santucci A. Angiotensin II stimulates intercellular adhesion molecule-1 (ICAM-1) expression by human vascular endothelial cells and increases soluble ICAM-1 release in vivo. Circulation 1999; 100: 1646-1652.

30 Liao TD, Yang XP, Liu YH, Shesely EG, Cavasin MA, Kuziel WA, Pagano PJ, Carretero $\mathrm{OA}$. Role of inflammation in the development of renal damage and dysfunction in angiotensin II-induced hypertension. Hypertension 2008; 52: 256-263.

31 Dzau VJ. Tissue angiotensin and pathobiology of vascular disease: a unifying hypothesis. Hypertension 2001; 37: 1047-1052. 